\title{
Fourphase Pulse Compression Sequences Generated using Chaotic Maps
}

\author{
K. Sridevi \\ Department of ECE, GIT \\ GITAM University \\ Visakhapatnam, India
}

\author{
J. B. Seventline \\ Department of ECE, GIT \\ GITAM University \\ Visakhapatnam, India
}

\author{
D. Elizabeth Rani \\ Department of EIE, GIT \\ GITAM University \\ Visakhapatnam, India
}

\begin{abstract}
Sequences with good correlation properties are in general required in various applications such as radar, sonar, spread spectrum and multiple access communication. The optimal sequences are the ones whose peak sidelobe of the aperiodic autocorrelation function is the minimum possible for a given sequence length. A chaotic sequence that provides auto and cross-correlation properties that are similar to those of random white noise is used for radar and spread spectrum systems. This paper presents a novel method of generating four phase codes applicable for radar pulse compression, which is derived from chaotic maps. It is also feasible to achieve simultaneously superior performances in detection range and range resolution using the proposed four phase coded sequences. The properties of these sequences like autocorrelation function, ambiguity function, Peak Sidelobe Ratio (PSR) and Integrated Sidelobe Ratio (ISR) have been studied. The properties of the codes generated by chaotic mapping method are very similar to those of the random four phase codes. The generation of these sequences is very simple, the available number of sequences is virtually infinite and number of sequences generated is not limited by the length of sequence.
\end{abstract}

\section{Keywords}

Pulse compression; peak sidelobe ratio; integrated sidelobe ratio; chaotic map;

\section{INTRODUCTION}

Pulse compression has been extensively used in operational radar systems since 1950 s to solve the practical problem of extending the operating radar range while maintaining the required range accuracy and resolution. It finds its importance in various fields like radar, sonar, seismic exploration and spread spectrum communication to achieve the high transmit energy of a long pulse while preserving the range resolution of a short pulse. Pulse compression thus allows the use of low power transmitters with longer pulse lengths to maintain the energy content of a pulse. A matched filter, the front end of the receiver is used to maximize the signal to noise ratio of the received signal. Various pulse compressed waveforms have been used for this purpose, including the linear frequency modulated signal, the nonlinear frequency modulated signal, biphase modulated sequences and polyphase modulated sequences [1-3]. Thecode or sequence is used to describe the phase of the individual sub pulses of a phase coded waveform [4]. Each type of coded waveform has certain desirable properties and the choice often depends on the application.

Theoretically, in pulse compression, the code is used to modulate the carrier waveform during transmission, which is used as the reference signal at the receiver. At the receiver front end, the reference signal is used to combine with the received signal to achieve a high range resolution. Phase coded waveforms divide the long pulse into $\mathrm{N}$ time segments, referred to as chips, and apply a different phase to each. A binary phase coded waveform limits the chip phase to 0 or 180 degrees, while polyphase coded waveform support more levels [5]. The binary sequences can be easily generated, processed and stored in digital circuitry. But the limitation comes when longer length sequences with lower Peak Sidelobe Ratio (PSR) are needed. The Peak Sidelobe Ratio is defined from the autocorrelation pattern as the ratio of the peak sidelobe amplitude to the mainlobe peak amplitude and is expressed in decibels. It was proved that the binary Barker sequences exist only up to a length of 13 with a PSR of $-22.3 \mathrm{~dB}$ [6]. But in some applications sequences with further reduction in PSR are required. To achieve this, Boehmer [7], Linder [8], Rao and Reddy [9] have obtained longer length binary codes violating the Barker conditions. But as the length of the sequence increases, the PSR did not reduce much. Therefore, it became much essential to switch over from biphase to fourphase sequences.

Though fourphase sequences do not have ideal energy efficiency like binary sequences, better sequences are available at larger lengths. The binary Barker sequences do not exist for length greater than 13, but four phase Barker sequences do exist at length 15. The generation of fourphase 15-bit generalized Barker sequence using acoustic surface waves is reported [10,11]. Richard J. Turyn proved that there are no even length Barker sequences of lengths $6,8,10,12,14$ and also that there are no new fourphase Barker sequences of length $\leq 31$ [12]. SAW devices are also used to generate fourphase sequences [13]. J. W. Taylor, Jr. generated good quadriphase or fourphase sequences using biphase to quadriphase transformation [14]. The advantages of these sequences are in the spectrum fall-off rate and the relative ease in which it lends itself to digital processing. Comparison with conventional pulse coding, in particular Barker's and Turyn'sbiphase sequences, the autocorrelation function is preserved when converted to fourphase sequences. The Doppler behavior of the quadriphase codes is proved by Taylor and Blinchikoff to be the same as the Doppler behavior of biphase codes. However, Levanon and Freedman has proved that the ambiguity diagram with a nonzero Doppler shift can be significantly different for a quadriphase code than that of the biphase code from which it was derived [15]. The ambiguity diagram of a quadriphase code derivedfrom a Barker code of length 13 has a diagonal ridge more like that of a linear-FM ambiguity function rather thanthethumbtack ambiguity function of the Barker code. But using these methods generation of fourphase sequences at large lengths has not been reported.

In this paper, a unique chaotic method of generating four phase radar signal having good correlation property is described. The use of chaotic sequences in spread spectrum communications is well documented [16]. A binary phase code generated using 
chaotic logistic map to achieve a low PSR was reported earlier [17]. Similar concept has been extended to the generation of good ternary sequences [21]. In that the ternary sequences were proved to exhibit superior behavior than binary sequences at all lengths. In this paper the generation of fourphase sequences using chaotic maps is discussed. Results show that the fourphase sequences perform better than binary and ternary sequences. The method discussed here has an advantage over the previous methods already available for fourphase sequence generation. Here, it is possible to generate sequences at larger lengths easily as it is not an exhaustive search method.

\section{CHAOTIC MAPS}

Chaos theory is a field of study in mathematics, having applications in several disciplines including physics, engineering, economics, biology and philosophy. Chaos theory explores the strange behaviour found in nonlinear deterministic dynamical systems that are highly sensitive to initial conditions. This effect is popularly referred to as the butterfly effect. A very small disturbance in initial condition produces a drastically different final solution for the chaotic system and the long-term prediction is not possible. This happens even though these systems are deterministic, meaning that their future behavior is fully determined by their initial conditions, with no random elements involved in this process. This behavior is known as deterministic chaos, or simply chaos [18]. Chaotic Sequences can be generated using different types of chaotic maps. Some of the maps are Henon Map, Lorenz Map, Logistic Map, Improved logistic map, quadratic map, Tent map and Cubic map. Chaotic generated sequences have also been considered for the implementation of CDMA systems. The greater number of sequences and their inherent security improvement have been considered as the main advantages of chaotic generated sequences. In this paper an attempt is made to generate fourphase sequences using logistic map, improved logistic map, quadratic map and Cubic map and their properties like PSR and ISR for different lengths are studied [19-20].

\subsection{The Logistic map}

The logistic map is a second order polynomial mapping, often considered as a prototype model for how complex, chaotic behaviour can arise from very simple non-linear dynamical equations.

$$
\text { The equation for logistic map is written as }
$$$$
x_{n+1}=f\left(x_{n}\right)=\mu x_{n}\left(1-x_{n}\right), \quad x_{n} \in(0,1)
$$

Where, the constant $\mu$ is called the bifurcation parameter with $\mu$ $\epsilon(0,4)$. The logistic equation is the basic example to illustrate the behaviour that is stable, periodic, and chaotic which depends on the value of $\mu$ in the equation. The series tends to a single value when $\mu$ is selected as $0<\mu<1$, independent of the initial value. With $\mu$ value between 1 and 3 , the series will approach the value $(\mu-1) / \mu$, independent of the initial value. The rate of convergence islinear, except for $\mu=3$. With $\mu$ value between 3 and 3.45 it can be observed that the system oscillates between 2 states for almost all initial conditions. With $\mu$ value between 3.5 and 3.54 the system oscillates between 4 states. When the system jumps between these states and it does not pass through any intermediary values. As the value of $\mu$ increases beyond 3.54 the number of states also increases to 8, 16 etc. This behavior is an example of a period-doubling cascade. The system undergoes increasingly frequent period doubling until it enters the chaotic regime at about 3.57. The system is in chaotic state when the value of $\mu$ is between 3.57 and 4 . Beyond $\mu=4$, the value of $x$ eventually leave the interval $(0,1)$ and diverge for almost all initial values.
Figure 1 shows the bifurcation diagram for the logistic map for all values of $\mu$ between 2.4 and 4 . The horizontal axis shows the values of the parameter $\mu$ while the vertical axis shows the possible long term values of $\mathrm{x}$ and it can be seen that the value of $\mathrm{x}$ is within the interval $(0,1)$ as long as the initial value is from the interval.

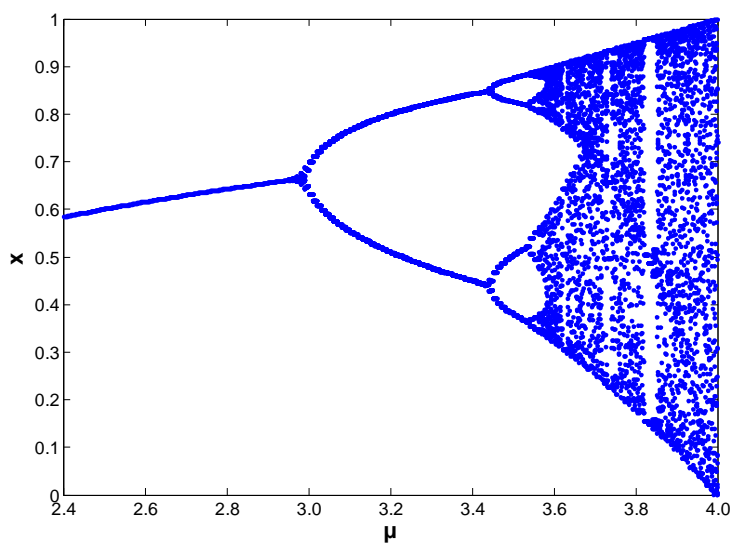

Fig1: Bifurcation diagram for the logistic map

The sequences generated using logistic map is inherently deterministic and a tiny disturbance in the initial condition $\mathrm{x}_{\mathrm{o}}$ gives rise to a tremendous difference in the outcome. This sensitive dependence can be illustrated by giving two very close initial points to the iterative map. After a few iterations, the two resulting sequences will look completely uncorrelated. Figure2 shows two such sequences for $\mu=4$ with $x_{0}=0.1$ and 0.10001 respectively. This small difference in the initial value leads to two completely different waveforms after a few iterations.

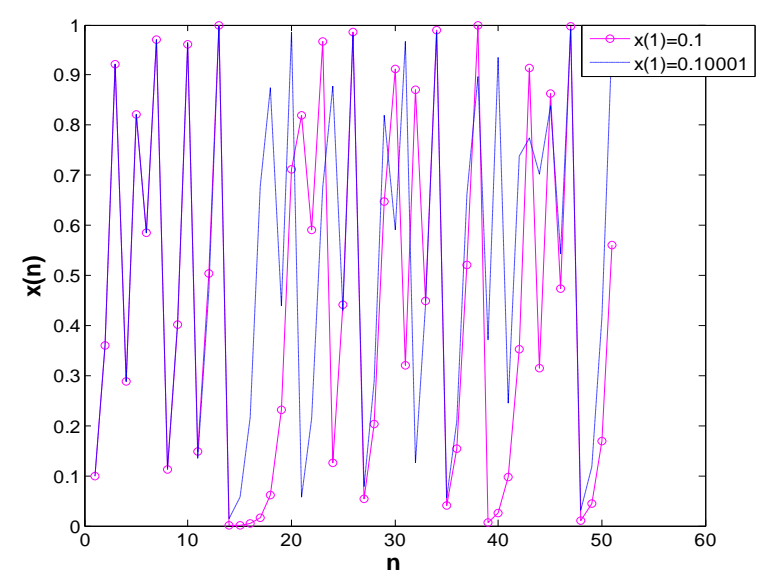

Fig 2: Chaotic behaviour of logistic map for $\mathrm{x}_{0}=0.1,0.10001$

\subsection{The Improved Logistic Map}

The improved logistic map is governed by the equation

$x_{n+1}=f\left(x_{n}\right)=1-2\left(x_{n}\right)^{2}, \quad x_{n} \in(-1,1)$

The chaotic sequence generated by improved logistic map is neither periodic nor converging and it is sensitively dependent on initial value. This equation shows chaotic behaviour for a initial value of $x_{0}$ varying from -1 to 1 . When the initial value $x_{0}$ is beyond this limit, $\mathrm{x}_{\mathrm{n}}$ tends to infinity as $\mathrm{n}$ tends to infinity. 


\subsection{The Cubic Map}

This map is described by the following equation

$x_{n+1}=f\left(x_{n}\right)=4\left(x_{n}\right)^{3}-3 x_{n}, \quad x_{n} \in(-1,1)(3)$

This equation also shows chaotic behaviour for an initial value of $\mathrm{x}_{0}$ varying from -1 to 1 like the improved logistic map. When $\mathrm{x}_{0}>1$ as $\mathrm{n}$ tends to infinity, $\mathrm{x}_{\mathrm{n}}$ also tends to infinity.

\subsection{The Quadratic Map}

The quadratic map is defined by the equation

$$
x_{n+1}=f\left(x_{n}\right)=0.5-4\left(x_{n}\right)^{2}, \quad x_{n} \in(-1,1)
$$

Like improved logistic map this equation shows chaotic behaviour for an initial value of $x_{0}$ varying from -1 to 1 . When $\mathrm{x}_{0}>1$ as $\mathrm{n}$ tends to infinity, $\mathrm{x}_{\mathrm{n}}$ also tends to infinity.

\section{GENERATION OF FOURPHASE SEQUENCES}

Even though there exists many methods for the generation of binary or fourphasesequences, they are either exhaustive search methods or they are techniques used to find only a finite number of sequences at a particular length. So, there is the chance of good sequences being neglected. Here, the method proposed for generation using chaotic maps has the advantage of generating virtually infinite number of sequences at any length. Thus this method has the advantage of finding almost all good sequences at a particular length. A Fourphase sequence usesfour alphabets for representation of data. A fourphase code is one whose subpulses are phased in one of four states: 0, 90, 180, 270 degrees; or equivalently $+1,+\mathrm{j},-1$, or $-\mathrm{j}$. The algorithm or method used for generating the fourphase sequence of length $\mathrm{N}$ using a chaotic map is given below

Step 1. Ensure that initially the chaotic map is in the chaotic region. For example, in logistic map the bifurcation parameter $\mu$ is selected to be 4 so that the map is in chaotic region.

Step 2. Select an initial value for $x_{n}$.

Step3. Using the chaotic map equations generate a raw sequence $\mathrm{x}_{\mathrm{n}}$ of length $\mathrm{N}$.

Step.4. The sequence is then quantized into four defined levels based on the threshold levels a, b and cas given in equation (5) to obtain the fourphase sequence $y_{n}$. The threshold levels were chosen randomly between minimum and maximum values of $\mathrm{x}$.For example, the $\mathrm{x}$ value varies between 0 and 1 for the logistic map. Therefore the threshold levels are chosen considering approximately the mean of the raw sequence obtained which is 0.5 in the case of logistic map. The level ' $a$ ' is chosen half of the mean of the raw sequence and level ' $c$ ' is chosen above the $3 / 4$ of the maximum value of the raw sequence, whereas the level ' $b$ ' is chosen as the mean value itself.

$$
\begin{aligned}
y_{n}=-1 \quad \text { if } x_{n}<a \\
=+j \quad \text { if } b \leq x_{n}<c \\
=+1 \text { if } x_{n} \geq c
\end{aligned}
$$

The various maps are extremely sensitive to the initial condition. By varying the initial condition $\mathrm{x}_{0}$, a totally uncorrelated fourphasesequence can be obtained. Therefore, the number of available sequences at a given length is virtually infinite.
Using the chaotic maps good fourphasesequences were generated for varied lengths using the above mentioned procedure. For logistic map the threshold levels $\mathrm{a}, \mathrm{b}$ and $\mathrm{c}$ is selected as $0.25,0.5$ and 0.75 respectively. The values of $a, b$ and $c$ are chosen as $-0.6,0$ and 0.6 for improved logistic map, $0.5,0$ and 0.4 for cubic map and $-0.25,0,0.25$ for quadratic map respectively. These levels are chosen after exhaustive searches of good sequences were done with different threshold levels. Thus the generation of sequences is simple, fast and reproducible. Using the above mentioned procedure fourphase sequences were generated for lengths from 10 to 5000 using the four chaotic maps. At every length the good sequences having a low value of PSR and ISR as defined by equation (7) and equation (8) are obtained. Several optimization techniques can be used to optimize the threshold levels, so that good sequences with good correlation properties can be obtained.

\section{PROPERTIES OF FOURPHASE SEQUENCES}

\subsection{Correlaton function}

The aperiodic autocorrelation $\mathrm{r}(\mathrm{k})$ of a sequence of length Nwhich is nothing but the output of the matched filter.Therefore the matched filter compressed output is givenby

$$
\begin{array}{r}
r(k)=\sum_{n=0}^{N-1-k} x_{n} x_{n+k}^{*}---- \\
\text { Where } k=-(N-1) \ldots .+(N-1)
\end{array}
$$

Theoretically, the autocorrelation characteristic of an infinitely long chaotic coded signal is nearly a Dirac Delta function. However, all the finite length sequences cannot be utilized due to rapid deterioration of correlation performance.

Thus the best sequences are selected based on the performance factor PSR. By varying the initial conditions a search for sequences with good autocorrelation pattern was done at every length for all the maps discussed above. The autocorrelation pattern of the good sequence generated using logistic map at length 5000 is shown in Fig.3. The figure has the shape of an impulse which is actually the desired response.

\subsection{Peak Sidelobe Ratio (PSR)}

One of the most commonly used performance measures is the Peak Sidelobe Ratio. The peak sidelobe is the largest sidelobe in the correlation of a sequence. The Peak Sidelobe Ratio is defined from the autocorrelation pattern $r(k)$ as the ratio of the maximum peak sidelobe amplitude to the mainlobe peak amplitude and is expressed in decibels. It is given by

$$
P S R=20 \log \frac{\operatorname{Max}|r(k)|}{r(0)} \text { where } k \neq 0 \text {---- }
$$

A sequence is defined as a good sequence if the PSR of the sequence is low. The reciprocal of PSR is known as the Discrimination Factor (D.F), which is expected to have a maximum value for better range detection. 


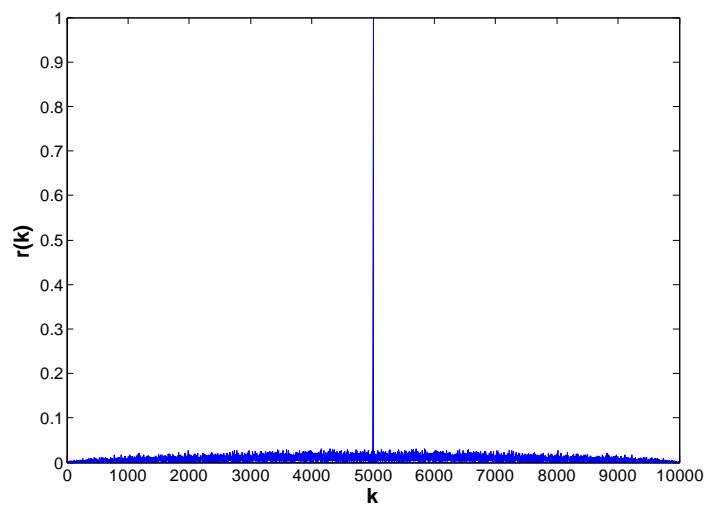

Fig 3:Normalized Autocorrelation pattern of logistic map of sequence length 5000

\subsection{Integrated Sidelobe ratio}

The Integrated Sidelobe Ratiois defined as the ratio of the total energy in the sidelobesto the energy in the main peak of the autocorrelation pattern and is expressed in decibels.

$$
I S R=10 \log \frac{2 \sum_{k=1}^{N-1} r^{2}(k)}{r^{2}(0)} \text { where } k \neq 0
$$

A sequence is defined as a good sequence if the ISR evaluated for that sequence is as minimum as possible.

Although the fourphase sequence generation process is quite general and easy by chaotic mapping methods, all sequences generated are not good or adequate for the pulse compression application in radar. So a complete search for good sequences has to be done by varying the initial conditions in each mapping method and from all the generated sequences, the best sequences are selected based on the PSR and ISR criterion defined by the equations (7) and (8) respectively. The Peak Sidelobe Ratio and Integrated Sidelobe Ratio obtained for good four phase sequences at different lengths for all the maps are tabulated in Table. 1 and Table.2. As observed from the Tables 1 and 2, the PSR decreases with the length of the sequence for logistic, improved logistic and quadratic maps. But, the PSR obtained with cubic map was found to decrease at lower lengths and increase at larger lengths. The variation of PSR as a function of sequence length for all the four chaotic maps can be clearly observed in the plot shown in Figure 4. From this plot it is clear that the three chaotic maps namely, the logistic map, the improved logistic map and the quadratic map work equally well at all lengths in the generation of good four phase sequences than the cubic map.

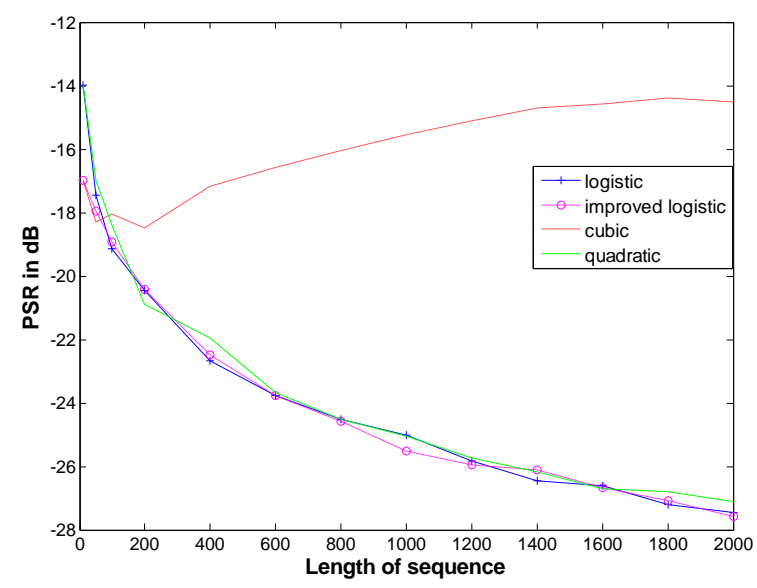

Fig4: PSR of fourphase sequences generated using Chaotic maps

Table 1.PSR and ISR variations of the fourphase sequences using logistic and improved logistic maps

\begin{tabular}{|c|c|c|c|c|}
\hline \multirow{2}{*}{$\begin{array}{l}\text { Length } \\
\text { of } \\
\text { sequence }\end{array}$} & \multicolumn{2}{|c|}{ Logistic map } & \multicolumn{2}{|c|}{ Improved logisticmap } \\
\hline & PSR in dB & ISR in $\mathbf{~} B$ & $\begin{array}{l}\text { PSR in } \\
\text { dB }\end{array}$ & ISR in $\mathbf{~ d B}$ \\
\hline 10 & -13.9794 & -4.6852 & -16.9897 & -5.8503 \\
\hline 20 & -16.4782 & -3.9794 & -16.4782 & -4.5593 \\
\hline 30 & -17.2379 & -3.6318 & -16.5321 & -3.2078 \\
\hline 40 & -17.4172 & -2.9243 & -17.4172 & -2.7573 \\
\hline 50 & -17.4473 & -2.1098 & -17.9588 & -3.1516 \\
\hline 60 & -18.4030 & -2.4243 & -18.3203 & -2.3326 \\
\hline 70 & -18.3286 & -2.0532 & -18.7728 & -2.8914 \\
\hline 80 & -18.7676 & -2.3331 & -18.1941 & -2.4270 \\
\hline 90 & -18.4403 & -1.8437 & -18.5541 & -1.9500 \\
\hline 100 & -19.1364 & -1.7289 & -18.9279 & -1.2587 \\
\hline 200 & -20.4455 & -1.1390 & -20.4335 & -1.4746 \\
\hline 300 & -21.6891 & -0.5392 & -21.6891 & -0.9004 \\
\hline 400 & -22.6660 & -0.9594 & -22.4940 & -0.3774 \\
\hline 500 & -23.3348 & -0.6607 & -23.1158 & -0.5364 \\
\hline 600 & -23.7618 & -0.5632 & -23.7646 & -0.2865 \\
\hline 700 & -24.2302 & -0.6677 & -23.8035 & -0.5109 \\
\hline 800 & -24.5265 & -0.3980 & -24.5710 & -0.4438 \\
\hline 900 & -24.9151 & -0.5260 & -24.8655 & -0.4722 \\
\hline 1000 & -25.0018 & -0.5608 & -25.5145 & -0.4312 \\
\hline 1500 & -26.4760 & -0.4061 & -26.5252 & -0.3337 \\
\hline 2000 & -27.4461 & -0.4450 & -27.5845 & -0.3434 \\
\hline 2500 & -28.1180 & -0.2294 & -28.1135 & -0.0976 \\
\hline 3000 & -28.7633 & -0.1679 & -28.7851 & -0.2955 \\
\hline 3500 & -29.2245 & -0.2568 & -29.5716 & -0.1854 \\
\hline 4000 & -29.7191 & -0.1427 & -29.6285 & -0.1583 \\
\hline 4500 & -30.1026 & -0.1958 & -30.1717 & -0.1641 \\
\hline 5000 & -30.5327 & -0.1988 & -30.1717 & -0.1960 \\
\hline
\end{tabular}


Table 2.PSR and ISR variations of the fourphase sequences using cubic and quadratic maps

\begin{tabular}{|c|c|c|c|c|}
\hline \multirow{2}{*}{$\begin{array}{l}\text { Length } \\
\text { of } \\
\text { sequence }\end{array}$} & \multicolumn{2}{|c|}{ Cubic map } & \multicolumn{2}{|c|}{ Quadratic map } \\
\hline & PSR in dB & $\begin{array}{c}\text { ISR in } \\
\mathrm{dB}\end{array}$ & $\begin{array}{c}\text { PSR in } \\
\text { dB }\end{array}$ & $\begin{array}{c}\text { ISR in } \\
\mathrm{dB}\end{array}$ \\
\hline 10 & -16.9897 & -5.8504 & -13.9794 & -4.6852 \\
\hline 20 & -16.4782 & -4.2022 & -16.4782 & -3.9794 \\
\hline 30 & -17.2379 & -3.3724 & -17.2379 & -3.6318 \\
\hline 40 & -16.9897 & -3.5902 & -17.4172 & -2.9243 \\
\hline 50 & -18.2974 & -2.7935 & -16.9897 & -1.6800 \\
\hline 60 & -17.4339 & -1.2752 & -17.9287 & -2.2185 \\
\hline 70 & -17.8711 & -2.5723 & -17.7638 & -1.7671 \\
\hline 80 & -18.0186 & -1.0272 & -18.1495 & -1.7393 \\
\hline 90 & -17.7495 & -1.4491 & -18.5541 & -1.7337 \\
\hline 100 & -18.0410 & -1.7941 & -18.3863 & -1.3525 \\
\hline 200 & -18.4695 & -1.0282 & -20.9018 & -1.3847 \\
\hline 300 & -18.0287 & 0.4400 & -21.6891 & -0.6461 \\
\hline 400 & -17.1769 & 0.5622 & -21.9680 & -1.0392 \\
\hline 500 & -17.2170 & 0.7514 & -23.3498 & -0.9909 \\
\hline 600 & -16.5703 & 0.1131 & -23.6709 & -0.4783 \\
\hline 700 & -15.8445 & 0.1802 & -23.9507 & -0.0455 \\
\hline 800 & -16.0337 & -0.1368 & -24.5035 & -0.5426 \\
\hline 900 & -15.9115 & 0.1320 & -24.5747 & -0.3385 \\
\hline 1000 & -15.5619 & 0.3254 & -25.0515 & -0.5088 \\
\hline 1500 & -14.5442 & 0.7812 & -26.2261 & -0.3840 \\
\hline 2000 & -14.5242 & 0.7143 & -27.1036 & -0.2863 \\
\hline 2500 & -14.2693 & 0.5901 & -27.9186 & -0.1295 \\
\hline 3000 & -13.9031 & 0.7415 & -28.4958 & -0.2024 \\
\hline 3500 & -13.7739 & 0.8742 & -29.0296 & -0.3253 \\
\hline 4000 & -13.5616 & 0.6852 & -29.5605 & -0.0111 \\
\hline 4500 & -13.4657 & 0.8932 & -30.1744 & -0.0307 \\
\hline 5000 & -13.5458 & 0.9144 & -30.4412 & -0.1379 \\
\hline
\end{tabular}

The plot of variation of ISR for the good sequences at lengths up to 2000 is shown in Figure 5. The ISR of the good sequences is not found to be satisfactory especially at higher lengths. From the plot it is understood that the ISR of the sequences generated using logistic map, improved logistic map and quadratic map is better than that of cubic map.

Now, the performance of four phase sequences is compared with that of binary and ternary sequences generated using the logistic mapping method $[17,21]$. The comparison plot of PSR of the binary, ternary and fourphase sequences are shown in Figure 6. The PSR of the four phase sequence decreases as the length of the sequence increases. It is found that at lower lengths up to 50, the PSR of ternary sequences is better than the binary and four phase sequences. But beyond length 50, the four phase sequences perform well compared to the binary and ternary sequences. As the chaotic sequence generation at higher lengths is easier, the four phase codes performance is superior compared to other codes at higher lengths.

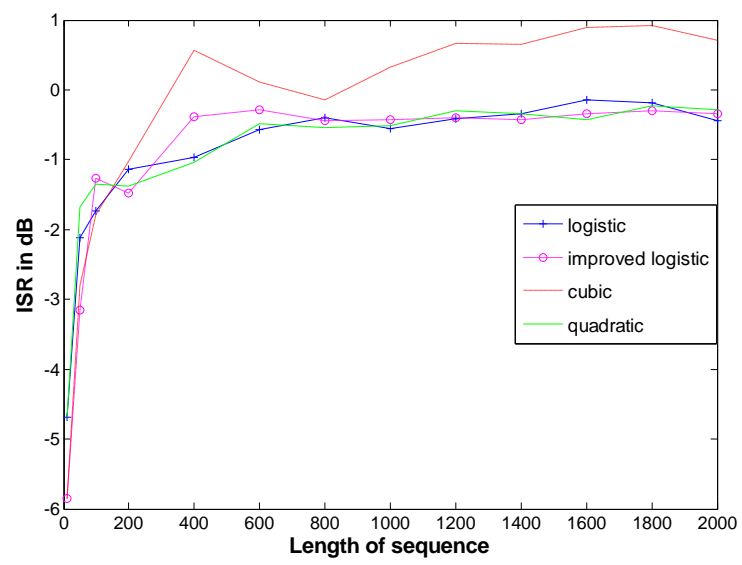

Fig 5: ISR of fourphase sequences generated using chaotic maps

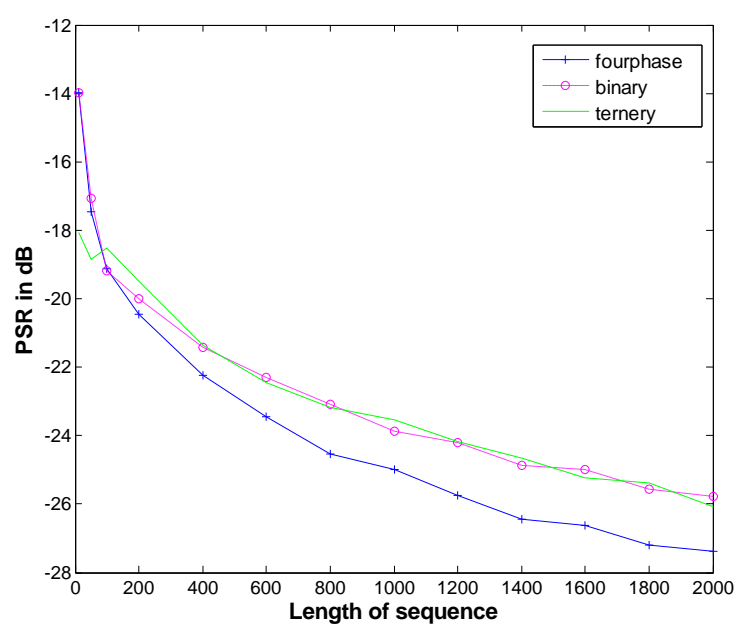

Fig6: Plot of PSR of binary, ternary and fourphase sequencesgenerated using logistic map

\section{AMBIGUITY FUNCTION}

The ambiguity function describes the complex envelope of the signal at the output of the radar receiver as a function of radar target range and radial velocity. The ambiguity function describes the output of a matched filter when the input signal is delayed by $\tau$ and Doppler shifted by $v$ relativeto nominal values for which the matched filter was designed. The ambiguity function is defined as

$$
\chi(\tau, v)=\left|\int_{-\infty}^{\infty} u(t) u^{*}(t+\tau) \exp (j 2 \pi v t) d t\right|
$$

$\mathrm{u}(\mathrm{t})$ represents the envelop of the fourphase coded waveform. The time delay is indicated by $\tau$ and $\mathrm{v}$ is the radial velocity depends on Doppler frequency. The ambiguity diagram of a 100 length fourphase sequence generated using logistic chaotic map is shown in figure 7. The ambiguity diagram approaches a thumbtack shape. The resolution of the waveform depends on the width of the spike centered at $|x(0,0)|$. As the width of the spike is very less, two closely spaced targets can be resolved easily both in range and velocity. 


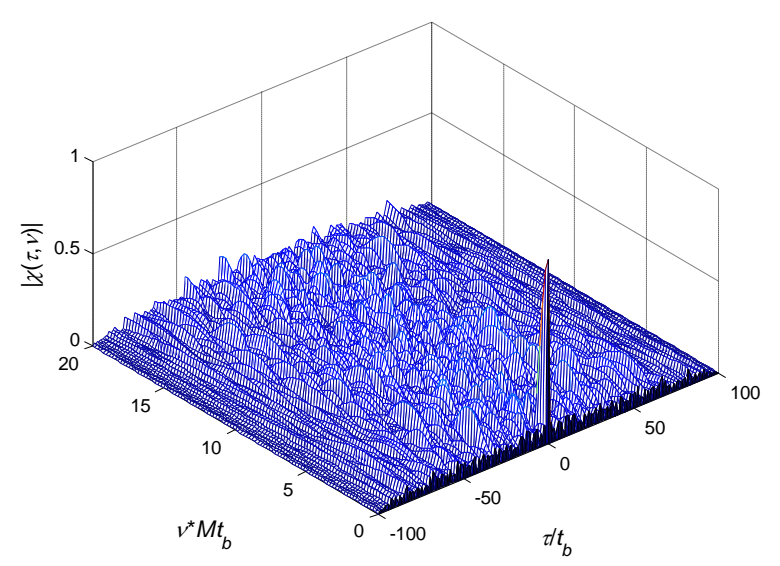

Fig7: Ambiguity diagram of 100 length fourphase sequence

\section{CONCLUSION}

Good four phase sequences are generated using logistic, improved logistic, quadratic and chaotic mapping method. The optimal codes are selected at various lengths based on the minimum Peak Sidelobe Ratio according to the performance requirements of the radar system. At larger lengths these codes offered very low PSR which is advantageous for the radar applications and spread spectrum communications. For lengths equal to and greater than 3000, PSR less than $-28 \mathrm{~dB}$ was obtained. These codes are able to achieve desired detection range and range resolution. Further improvement in performance can be achieved by applying optimization techniques for the three parameters namely the initial value $\mathrm{x}_{0}$ of this mapping, the incremental value and the quantization levels and better sequences can be obtained.

\section{REFERENCES}

[1] Cook, C. E. and Bernfield, M. 1967,Radar Signals - An Introduction to Theory and Application.

[2] Merrill I. Skolnik 2002, Introduction to Radar System. $\left({ }^{3 r d}\right.$ ed.)

[3] Nathanson, F.E., Reilly, J.P. and Cohen, M. 1991 "Radar Design Principles ( $2^{\text {nd }}$ ed.)".

[4] Kerdock, A.M., Mayer,R. and Bass,D. 1986,Longest Binary Pulse compression codes with given peak sidelobe levels,Proceedings of IEEE, vol.74, No.2, pp.366.

[5] Levanon, N. and Mozeson, E. 2004.Radar Signals.

[6] Rao, B. V., and Deshpande, A. A. 1998,"Why the barker sequence bit length does not exceed thirteen", Journal of IETE, vol. 34, no. 6, pp. 461-462.

[7] Boehmer, A. M., 1967,"Binary pulse compression codes", IEEE Trans.IT-13, no. 2, pp. 156-157.
[8] Linder, J.,1975, "Binary sequences upto length 40 with best possible autocorrelation function", Electronic Letters, vol. 11, no.21, pp. 507-508.

[9] VeerabhadraRao, K., Umapathy Reddy,1986, "Biphasesequences with low sidelobe autocorrelation function", IEEETransactionsonAerospace and Electronic Systems, vol. 22, no. 2, pp. 128-133.

[10]Golomb, S.W. and Scholtz, R.A., 1965, "Generalized Barker Sequences", IEEE Trans. On Inform. Theory,vol. IT-11, pp. 533- 537.

[11]Van De Vaart, H., 1972, "Generation and Correlation of Quadriphase Coded signals using Acoustic Surface Wave”, Proc. Letters of IEEE, pp. 454- 455.

[12] Richard, J. Turyn, 1974, “Four-Phase Barker Codes”, IEEE Trans. On Inform. Theory, vol. IT-20, n0.3, pp. 366- 371.

[13]Vale, C. R., 1981, “SAW quadriphase code generator”, IEEE Trans. On Microwave Theory and Techniques, MTT-29, pp. $410-414$.

[14] Taylor, J. W., Jr. and Blinchikoff, H. J., 1988, "Quadriphase code-A Radar Pulse Compression Signal With Unique Characteristics", IEEE Trans. On Aerospace and Electronic systems, vol. 24, n0.2, pp. 156- 170.

[15] Levanon, N. and Freedman, A., 1989,“Ambiguity Function of Quadriphase coded Radar Pulse", IEEE Trans. On Aerospace and Electronic systems, vol. 25, n0.6, pp. 848853.

[16] Heidari-Bateni, G. andMcgillen, C. D., 1992,"Chaotic sequences for spread spectrum: An alternative to $\mathrm{PN}$ sequences". IEEE, ICSTWC, pp. 437-440.

[17]Xin Wu, Weixian Liu, Lei Zhao and Jeffrey, S. 2001,"Chaotic phase code for radar pulse compression",Proceedings of IEEE NationalRadar Conference, Atlanta (USA), pp. 279-283.

[18] Devaney, R.L.1992, A first course in chaotic dynamical systems: Theory and experiment.

[19] Ashtari, A., Thomas, G. and Flores, B. C., 2007,"Radar signal design using chaotic signals". Proceedings of International WaveformDesign and Diversity Conference, Pisa (Italy), pp. 353-357.

[20] Hilborn, R. C., 1994,Chaos and Nonlinear Dynamics: An Introduction for Scientists and Engineers.

21] Seventline, J.B., Elizabath Rani, D. andRajarajeswari, K., 2010, "Ternary Chaotic Pulse Compression sequences", Radio Engineering, vol. 19, no.3, pp. 415-420. 